\title{
Neutrinos from GRBs, and the connection to gamma-ray observations
}

\author{
Philipp Baerwald*, Svenja Hümmer, and Walter Winter \\ Universität Würzburg, Germany \\ E-mail: philipp.baerwaldephysik.uni-wuerzburg.de, \\ svenja.huemmer@physik. uni-wuerzburg.de, \\ winterephysik.uni-wuerzburg.de
}

\begin{abstract}
The possibility of GRBs as the source of UHECR is currently starting to get constrained by the non-observation of GRB neutrinos. We therefore reconsider the current neutrino flux predictions from gamma-ray observations, based on the internal shock model, from the particle physics involved, and numerically calculate the resulting spectra. At the example of the Waxman-Bahcall GRB neutrino flux we demonstrate how additional production modes, synchrotron cooling of secondary particles, and flavor mixing affect the expected neutrino spectrum. We demonstrate that a detailed (numerical) calculation changes the prediction of the GRB neutrino flux from gamma-ray observations, apply this to the recent IC40 GRB results, and discuss systematical and astrophysical uncertainties of this interpretation.
\end{abstract}

Gamma-Ray Bursts 2012 Conference-GRB2012,

May 07-11, 2012

Munich, Germany

\footnotetext{
* Speaker.
} 


\section{Introduction}

In the 100 years since the discovery of cosmic rays by R. Hess there have been several measurements confirming the extent of the cosmic ray spectrum to highest energies.However the source of the ultra-high energy cosmic rays (UHECR) so far remains a mystery. Due to the deficiencies of $\mathrm{CR}$ when it comes to tracing their sources other means of detecting proton acceleration to the highest energies have been proposed. Especially the detection of a neutrino signal associated to the UHE protons would be a smoking gun for UHECR production. Here, gamma-ray bursts (GRB) are one of the prime candidates as possible UHECR sources since models predict a distinct neutrino single from these events [1]. The prediction together with the timing information should give flux limits which are testable by $\mathrm{km}^{3}$ neutrino telescopes, such as IceCube.However, since IceCube is finally completed and the first (negative) results are being published, see Refs. [2, 3], it is time to revisit the original models.

In the first section we will revisit the standard approach used for the GRB analyses and explain the multi messenger approach used for the flux predictions. Then, in the second section, we will explain how the normalization of the flux changes due to corrections to the analytical approach and how a detailed particle physics treatment changes the shape of the predicted neutrino spectrum. Finally, we will apply this new prediction method to the bursts from the IceCube40 analysis [2] and show how the prediction changes the interpretation of current results.

\section{Modeling neutrinos from GRB}

Predictions of neutrino fluxes are mostly done for the fireball model with the prompt emission coming from internal shocks. The shocks are assumed to accelerate charged particles such as protons via Fermi acceleration to ultra-high energies as well as give rise to the prompt gamma-ray emission. If these high energy protons and photons interact neutrinos can be produced. The most commonly used approximation for these photohadronic interactions is the $\Delta$-resonance approximation as suggested by Waxman and Bahcall [1]

$$
p+\gamma \rightarrow \Delta^{+} \rightarrow\left\{\begin{array}{ll}
n+\pi^{+} & 1 / 2 \text { of all cases } \\
p+\pi^{0} & 1 / 2 \text { of all cases }
\end{array} .\right.
$$

In the subsequent decay of $\pi^{+}$into muons and then positrons a total of three neutrinos is produced,

$$
\begin{aligned}
\pi^{+} \rightarrow & \mu^{+}+v_{\mu}, \\
& \mu^{+} \rightarrow e^{+}+v_{e}+\bar{v}_{\mu} .
\end{aligned}
$$

Moreover, using the observed photon spectrum as target spectrum and the particle physics involved in the photohadronic interactions it is possible to also give predictions on the shape of the GRB neutrino flux. The observed spectrum of a GRB is assumed to be a broken power law with a spectral index $\alpha_{\gamma} \simeq 1$ below the break energy $\varepsilon_{\gamma \text {,break }}$ and a spectral index $\beta_{\gamma} \simeq 2$ above. Moreover it is assumed that the shock accelerated protons follow a spectrum $f_{p}(E) \propto E^{-2}$. Combining these spectra it is reasoned that the neutrino spectrum should have a "lower" break at $E_{v, b}$ (from break in photon spectrum) and a "higher" break at $E_{v, \pi}$ (from synchrotron losses of secondary particles), 
see e.g. Ref. [4]. This leads to a characteristic flat plateau shape in the $E^{2} \frac{\mathrm{d} N_{v}}{\mathrm{~d} E_{v}}$ over $E$ plot called the Waxman-Bahcall flux shape,

$$
E_{v}^{2} \frac{\mathrm{d} N_{v}}{\mathrm{~d} E_{v}}=C_{v} \cdot \begin{cases}\left(E_{v} / E_{v, b}\right)^{-\alpha_{v}} & \text { for } E_{v}<E_{v, b} \\ \left(E_{v} / E_{v, b}\right)^{-\beta_{v}} & \text { for } E_{v, b} \leq E_{v}<E_{v, \pi} \\ \left(E_{v} / E_{v, b}\right)^{-\beta_{v}}\left(E_{v} / E_{v, \pi}\right)^{-2} & \text { for } E_{v} \geq E_{v, \pi}\end{cases}
$$

with the spectral indices $\alpha_{v}=\beta_{\gamma}-1 \simeq 1$ and $\beta_{v}=\alpha_{\gamma}-1 \simeq 0$ as well as the normalization $C_{v}$.

The normalization $C_{V}$ can either be calculated by using the cosmic ray data to obtain a collective normalization for all bursts, see for example Refs. [5, 6], or by using photon data from individual bursts and stacking them to obtain a diffuse flux, see appendices of Refs. [4, 2]. The latter approach is used for the current IceCube limits by calculating the expected flux based on the observed GRB. The model used by IceCube [2] (IC-FC), based on Ref. [4], relates the observed photon spectrum to the neutrino spectrum via the energy in protons.

$$
\int_{0}^{\infty} \mathrm{d} E_{v} E_{v} F_{v}\left(E_{v}\right)=\overbrace{\frac{1}{8}}^{x_{\pi \rightarrow v}=\frac{1}{2} \cdot \frac{1}{4}} \underbrace{\left(1-\left(1-\left\langle x_{p \rightarrow \pi}\right\rangle\right)^{\Delta R / \lambda_{p \gamma}}\right)}_{f_{\pi}} \overbrace{\frac{1}{f_{e}} \int_{1 \mathrm{keV}}^{10 \mathrm{MeV}} \mathrm{d} E_{\gamma} E_{\gamma} F_{\gamma}\left(E_{\gamma}\right)}^{\text {energy in protons }}
$$

A recent analysis with this approach showed that the expected neutrino flux is already so high that there should have been a signal in IceCube, see Ref. [3]. The question at this point is if this really rules out GRB as source of UHECR or if the more detailed current models give a different result. In the next section we will show how the prediction changes when one reanalyzes the steps of the approach described in this section.

\section{Changes to the prediction model}

When the result of the IC-FC is compared to the numerical fireball calculation (NFC) from Ref. [7] there is a significant difference in the predictions, see left plot of Fig. 1. This change can be pinned down to two types of effects: corrections to the analytical model and effects of the detailed particle physics treatment.

In the right plot of Fig. 1 you can see the effect of the (analytic) revised fireball model prediction at the example of GRB080603A. The first significant change is that the spectrum should have two breaks due to synchrotron losses, one at $E_{v, \pi}$ for neutrinos from pions and one at $E_{v, \mu}$ for those from muons. Moreover, the first break shifts due to a correction to the threshold energy of the photohadronic interactions, see [8], and there is an additional shift from considering the cosmic expansion. The second change is the corrected normalization which consists of two parts: $c_{S}$ corrects for the energy losses of the secondary particles leading to different spectral shapes and the energy dependent mean free path of the protons, and $c_{f_{\pi}}$ is a correction to the pion production efficiency. The latter corrects for the incorrect usage of the peak photon energy as average photon energy $\left(f_{C_{\gamma}}\right)$, a rounding error in the old formulas $\left(f_{\approx}=0.69\right)$, and for neglecting the width of the $\Delta$-resonance $\left(f_{\sigma} \simeq 2 / 3\right)$. Depending on the parameters of the burst this can reduce the flux by over an order of magnitude. For more details please refer to Ref. [7].

Moreover, numerical calculations as in Ref. [8] have shown that there are several additional effects 
when the detailed particle physics involved are considered. Apart from the $\Delta$-resonance there are also contributions from higher resonances, direct ( $t$-channel) production, and high energy (multi pion) processes to the full photohadronic interaction cross section. Hence, also $\pi^{-}, n$, and heavier particles such as $\mathrm{K}^{+}$need to be considered in the decays. Additionally the intermediate particles need to be treated individually to correctly include the energy losses of secondary particles. Furthermore helicity dependent muon decay and flavor mixing of neutrinos is considered. This partially counters the reduction of the flux and again changes the flux shape, see curve labeled NFC in left plot of Fig. 1. With this revised approach it is possible to recalculate the neutrino flux prediction from the IceCube40 analysis.
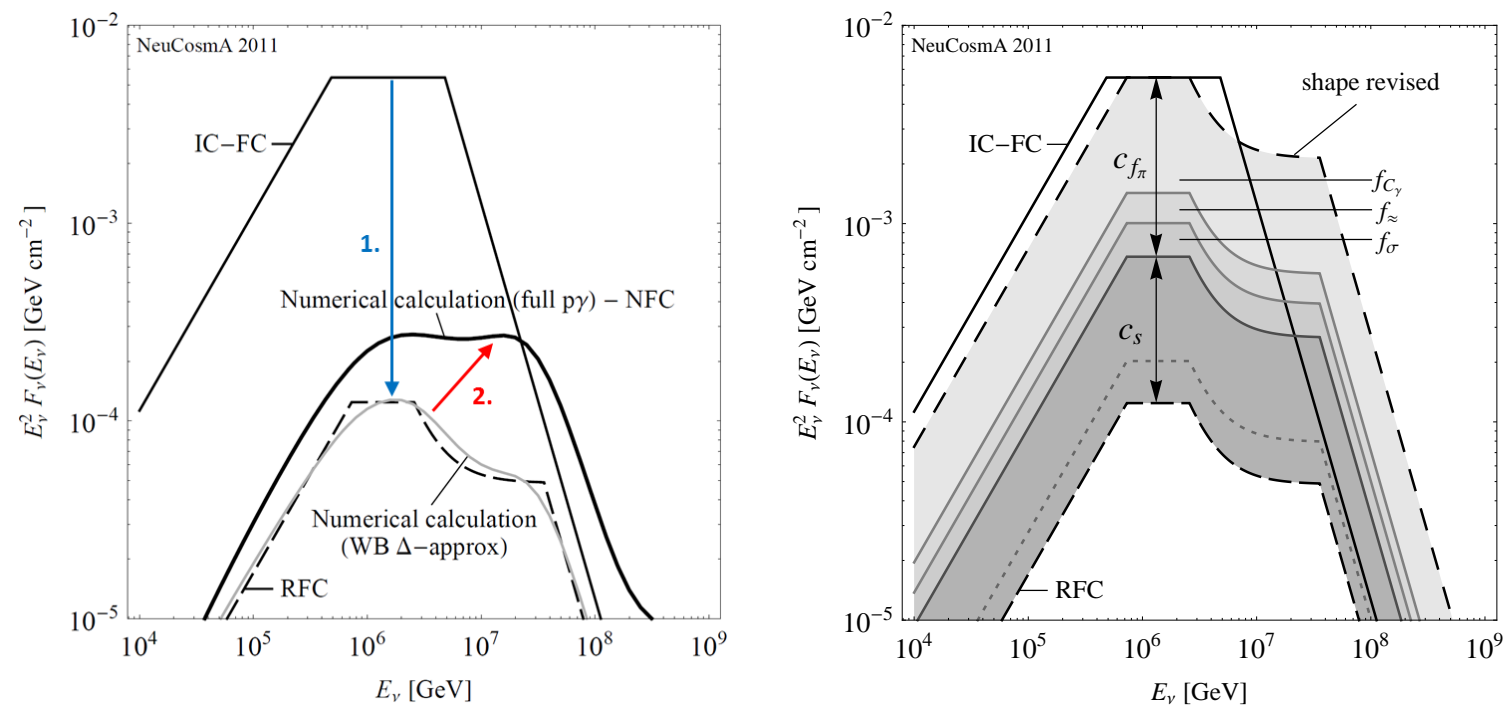

Figure 1: The left plot shows the two steps of the change of the analytic model from Ref. [2] (IC-FC) to the numerical calculation from Ref. [7] (NFC). In the first step the analytic model is revised leading to a lower normalization and slightly changed flux shape (RFC). In the second step the effect of the detailed particle physics treatment photohadronic interactions enhances the flux again and changes the flux shape to a double peak shape. In the right plot the different contributions leading to the significantly reduced flux from the revised analytical calculation (RFC) compared to the IceCube prediction (IC-FC) are depicted. Both figures adopted from Ref. [7].

\section{IceCube40 reanalysis}

The IceCube40 GRB analysis is based on 117 bursts detected between April 2008 and May 2009 which are then used to extrapolate a diffuse flux [2]. With the data available for each burst it is possible to sample the expected neutrino flux on a burst-by-burst basis. Using the same parameters for each burst and the numerical approach described in the previous section it is possible to recalculate the neutrino flux [7]. As can be seen from left plot of Fig. 2 the resulting diffuse flux prediction is roughly an order of magnitude below the original IC40 prediction. Moreover, the nominal prediction is still below the sensitivity of IC40+59. However, the result strongly depends on the chosen burst parameters. If one varies the standard values used for unknown parameters the 
flux prediction can greatly vary, marked "astrophysical uncertainties" in left plot of Fig. 2. Additionally there is the statistical uncertainty from extrapolation from a small number of bursts to a diffuse flux limit.

Furthermore it needs to be considered that the size of the uncertainties is model dependent, as can be seen from the right plot of Fig. 2. Here, the uncertainty on the IceCube prediction is taken from Fig. 3 of Ref. [3] and based on a specific model that assumes that the target photons are created by synchrotron radiation of electrons. Compared to newer predictions such as from Ref. [9] or the previously discussed one from Ref. [7] these rather small uncertainties seem rather optimistic. While He et al. consider the emission radius as a free parameter and also include possibilities of other GRB models such as a dissipative photosphere or magnetic reconnection leading to a very large uncertainty on the flux level, Hümmer et al. keep the target photons fixed to the observed spectrum and only vary the burst parameters. The actual uncertainty ranges are taken from Fig. 3 of Ref. [9] and Fig. 3 of Ref. [7], respectively. It shows that both newer predictions give a lower result for the flux prediction, however the uncertainties as well as the nominal result are highly model dependent. Nevertheless, even for the fireball model with prompt emission from internal shocks the parameter space is already starting to get limited from the IceCube results. With more data these constraints will get even stronger and the whole search is more like the search for a new particle such as the Higgs search at LHC. There could be a discovery of a signal in neutrinos during the next few years or the parameter space could be constrained to the point where this fireball model with internal shocks is mostly ruled out.
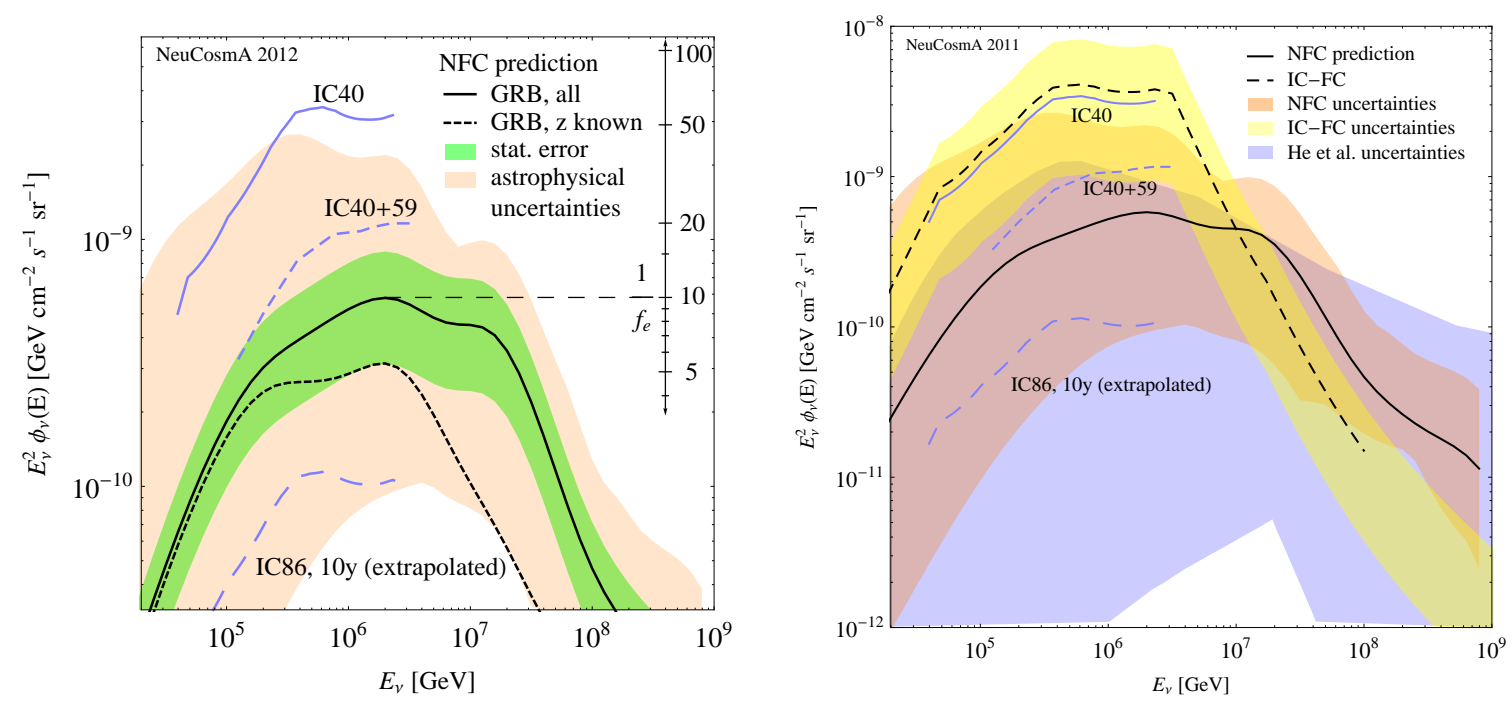

Figure 2: The left plot shows the recalculation of the diffuse GRB neutrino flux based on the IceCube 40 GRB sample [2]. The nominal prediction is still below the current IC40+59 sensitivity [3]. However, as depicted by the shaded areas there are large uncertainties due to the (unknown) GRB parameters (peach colored area) and statistical uncertainties (green area), taken from Ref. [7]. The right plot shows the uncertainty ranges for three different flux predictions, namely the IceCube40 prediction [2] together with the uncertainty taken from Fig. 3 of Ref. [3], the range given in Fig. 3 of Ref. [9], and the flux prediction and uncertainty from Fig. 3 of Ref. [7]. 


\section{Summary}

It has been shown that the neutrino flux can be predicted on a burst-by-burst basis from the observed photon spectra. Moreover, the computation of the flux shape as well as the normalization have been reanalyzed and several factors which change the older predictions could be pin-pointed. These factors correcting the normalization all point in the same direction, leading to a significantly lower flux prediction. This is partially countered when using the full photohadronic interaction cross section instead of the $\Delta$-resonance approximation. In the full numerical calculation further production modes of neutrinos, individual energy losses of the intermediate particles, helicity dependent muon decay, and flavor mixing of the neutrinos were included as additional effects. This detailed particle physics treatment alters the predicted flux shape to be a double peak structure with an additional high energy component from kaon decay instead of the famous WB plateau shape. When this revised model was applied to the 117 bursts of the IC40 GRB analysis the nominal prediction was still below the current IceCube sensitivity. However, the uncertainties on the parameters of the individual GRBs lead to a large error on the flux prediction. Therefore a more accurate assessment of the current IceCube results should be that IceCube is currently cutting into the accessible parameter space of this GRB model. With about 10 years of data taking with the full detector it should be possible to either exclude most of the parameter space and mostly rule out the specific internal shock fireball model, or see the first neutrinos from GRB.

Acknowledgements: P.B. is supported by the DFG Graduiertenkolleg GRK1147.

\section{References}

[1] E. Waxman and J. N. Bahcall, High-energy neutrinos from cosmological gamma-ray burst fireballs, Phys.Rev.Lett. 78 (1997) 2292-2295, [astro-ph/9701231].

[2] IceCube Collaboration Collaboration, R. Abbasi et al., Search for muon neutrinos from Gamma-Ray Bursts with the IceCube neutrino telescope, Astrophys. J. 710 (2010) 346-359, [arXiv:0907.2227].

[3] IceCube Collaboration Collaboration, R. Abbasi et al., An absence of neutrinos associated with cosmic-ray acceleration in $\gamma$-ray bursts, Nature 484 (2012) 351-353, [arXiv: 1204 .4219].

[4] D. Guetta et al., Neutrinos from individual gamma-ray bursts in the BATSE catalog, Astropart. Phys. 20 (2004) 429-455, [astro-ph/ 0302524 ].

[5] E. Waxman and J. N. Bahcall, High energy neutrinos from astrophysical sources: An upper bound, Phys. Rev. D59 (1999) 023002, [hep-ph/9807282].

[6] M. Ahlers, M. Gonzalez-Garcia, and F. Halzen, GRBs on probation: testing the UHE CR paradigm with Ice Cube, Astropart.Phys. 35 (2011) 87-94, [arXiv: 1103.3421 ].

[7] S. Hümmer, P. Baerwald, and W. Winter, Neutrino Emission from Gamma-Ray Burst Fireballs, Revised, arXiv:1112.1076.

[8] P. Baerwald, S. Hümmer, and W. Winter, Magnetic Field and Flavor Effects on the Gamma-Ray Burst Neutrino Flux, Phys. Rev. D83 (2011) 067303, [arXiv: 1009.4010 ].

[9] H.-N. He et al., Icecube non-detection of GRBs: Constraints on the fireball properties, arXiv:1204.0857. 